\title{
Two Parsing Algorithms by Means of Finite State Transducers
}

\author{
Emmanuel Roche* \\ Mitsubishi Blectric Research Laboratories \\ 201, Broadway, Cambridge, MA 02139, roche(ümerl.com
}

\begin{abstract}
We present a new approach, illustrated by two algo rithms, for parsing not only linite Slate (iranmars but also Context Free Grammars and their extension, by means of finite state machines. The basis is the computation of a fixed point of a finite-state function, i.e. a finite-state transducer. Using these technigues, we have built a prograrn that parses French sertiences with a grammar of more than 200,000 lexical rules with a typical response time of less than a second. The first algorithm computes a fixed point of a non-deterministic finite-state transducer and the second computes a fixed point of a deterministic bidirectional device called a bimachine. These two algorithms point out a new connection between the theory of parsing and the theory of representation of rational transductions.
\end{abstract}

\section{INTRODUCTTON}

Finite state devices have recently attracted a lot of interest in computational linguistics. Computational efficiency has been drastically improved for morphological analysis by representing large dictionaries with Pinite State Automata (FSA) and by representing twolevel rules and lexical information with finite-state transducers [8, 4] More recently, [11] has achieved parsing with low level lexical sensitivity by means of linite state automata. Finite state approxination of contextfree grammars also proved both useful and eflicient for certain application [9].

One common motivation of all this work is to improve efliciency drannatically, hoth in terus of time amd space. These results often provide programs orders of magnitude faster than more traditional implementations. Moreover, FSAs are a natural way to express lexical sensitivity, which has al ways been a requirement in morphology and which laas proved crucial in syntax. The granmar we used for French, called LexiconCirammar (sce [6] [7] [2] [3] [10] for instance), pustres the lexicalization very far and it is our belief that this lexicalization trend will amplify itself and that it will result in grammars several orders of magnitude larger than today's representations. This uncovers the need for new methods that will be able to handle such large scale grammars.

"Supported by a DRLT-Fcole P'olytechnique contract, this work was done at the Institut Gispsard Monge and at the LADL.
However, a main drawback of the finite state approach to synlax is the difliculty of representing hierarchical data; this partly explains why PSA-based programs only do incomplete parsing. 'This paper presents a new parsing approach based on finite-state transducers, a device that has been used alrealy in morphology [8] but not yot in syntax, that provides both hierarchical representations and efficiency in a simple and nalural way. The representation is very compact, this allows to implement large lexical grammars.

'Two new parsing algorithms illustrate the approach presented here. 'Tlhe first one uses a linite state transducer and compules a fixed point. But finite state transelucers, mblike SSAs, cannot be made deterministic; however, a hidirectional device called a himachine [1] can indirectly make them deterministic. 'This leads to the second algrorithm presented here. The very high efleciency of this approach can be seen in the experiments on french. Sentences can be parsed with a grammar containing more than 200,000 lexical rules'; this grammar is, we think, the largest grammiar ever implemented.

\section{PRINCIPLES}

The concept of Finite-State Transducer

The basic concept here, since we nol only match but also add markers, is the concept of finite-state transducer. 'This device has already proved very eflicient in morphological analysis [8]. It can deal wilh very latges anount of data, namely morphological dielionaries containing more than 500,000 entries.

$A$ linite state transducer is simply an PSA except that, white following a path, symbols are enitted. A finite state transducer can also simply be seen as a graph where the vertices, called states, are linked through oriented arrows, called transitions. 'The transitions are labeled by pairs (inpul_label, oulpuldabel) ${ }^{2}$.

\footnotetext{
'By lexical rule we basically mean a sentence struchure, as for example: Nhum say to Nhutn that $S$, where Nhum and $S$ respeclively stand for luman nominal and sentence. Thus the rules we deal with can roughly be seen as sentence structures where at least one element is lexical. This will be developed in section

${ }^{2}$ An extensive description of this concept can be lound in [1].
} 


\section{The parser in term of rational transduction}

In our parser, the grammar is a rational transduction $f$, represented by a transducer $T$. The input of the parser is the set $s_{0}$ containing as only element the input sentence bounded by the phrase marker $[P]$, i.e. $s_{0}=\{[P]$ sentence $[P]\}$. The analysis consists in computing $s_{1}=f\left(s_{0}\right), s_{2}=f\left(s_{1}\right)$ until a fixed point is reached, i.e. $s_{p}=f\left(s_{p}\right)$. The set $s_{p}$ contains trees represented by bracketed strings, this set is the set of grammatical analysis of the sentence, it contains more than one element in the case of syntactically ambiguous inputs. Each set $s_{i}$ is represented by a Directed Acyclic Graph (DAG) $A_{i}$, thus the computation consists in applying the transducer $T^{\prime}$ on the DACs $A_{i}$. We shall write it $A_{i+1}=T\left(A_{i}\right)$.

In the next section we give two complete examples of that.

\section{TWO SIMPLE EXAMPLES}

An example of a Top-Down analysis

The graph on figure 1 describes the analysis of the sentence:

$$
s_{1}=\text { John said that Mary left }
$$

The graph on this figure has to be read in the following way: the input sentence is represented by the DAG $A_{1}$ on the upper left corner; the subset of the grammar required for the analysis of this sentence is the transducer $\int$ on the right hand side of the figure 1.

The analysis is then computed in the following way: we apply the transducer $f$ to $A_{1}$, that is we compute $A_{2}=f\left(A_{1}\right)$, this represents one step of a Top-Down analysis of the sentence. The box with a star inside represents this operation, namely applying a transducer to a $\mathrm{DAC}$. If we then apply $f$ to this result (i.c. $A_{2}$ ), we obtain $A_{3}=f\left(A_{2}\right)=f^{2}\left(A_{1}\right)$ represented under $A_{2}$. If this operation is applied once more, one gets $A_{4}=f\left(A_{3}\right)=f^{3}\left(A_{1}\right)$. This last result, $A_{4}$, is a fixed point of the transducer $f$, i.e. $f\left(A_{4}\right)=A_{4} . A_{4}$ is a DAC: that represents a finite set $\operatorname{Set}\left(A_{4}\right)$ of strings. Here, this set only contains one element, namely Set $\left(A_{A}\right)=$ $\{(\mathrm{John}) N 0($ said $)$ V0(that (Mary) N0(lefl)V0)ThatS $\}$. Each element is a bracketed representation of an allatysis. Here the analysis is unique.

\section{An example of a simultaneous Top-Down Bottom- $U$ p analysis}

The previous example might give the impression that computing a fixed point of a transducer automatically leads to simulating a top-lown context free analysis. However, we shall now see that using the flexibility of manipulating transclucers, namely being able to compute the composition and the union of two transducers, allows a context sensitive parsing which is simultaneously Top-Down and Bottom-up with the possibility of choosing which kind of rule should be parsed BottomUp.

Suppose one wants to analyze the sentence $s_{2}=$ Max bought a little bit more than five hundred share certificates. Suppose one has the following small functions, each one being specialized in the analysis of an atomic fact (i.e. each function is a lexical rule):
- $f_{1}: w$ a little bit more than $w^{\prime} \longrightarrow w$ (pred a little bit more than pred) $w^{\prime} ; w, w^{\prime} \in A^{*}$

- $\int_{2}{ }^{3}: w$ five hundred $w^{\prime} \longrightarrow w$ (num five hundred num) $w^{\prime}$

where $w \in A^{*}$ and $w^{\prime} \in A^{*}-\{N U M E R A L\}$

- Sa: $w$ share certificates $w^{\prime} \rightarrow w$ (on share certificates cn) $w^{\prime}$ where $w, w^{\prime} \in A^{*}$

- $f_{4}:[\mathbf{P}] w$ bought $w^{\prime}[\mathbf{P}] \longrightarrow[\mathbf{N} w \mathbf{N}]$ bought $[\mathrm{N}$ $\left.w^{\prime} \mathrm{N}\right]$ where $w, w^{\prime} \in A^{*}$

- $\int_{5}: w[\mathrm{~N} \operatorname{Max} \mathrm{N}] w^{\prime} \longrightarrow w \operatorname{Max} w^{\prime} ; w, w^{\prime} \in A^{*}$

- $\int_{6}: w_{1}\left[N\right.$ (prod $w_{2}$ pred) (num $w_{3}$ num $)$ (cn $w_{4}$ cn $\left.) \mathbf{N}\right] w_{5} \longrightarrow w_{1}\left(\mathrm{~N} w_{2} w_{3} w_{4} \mathbf{N}\right) w_{5}$

where $w_{1}, w_{2}, w_{3}, w_{4}, w_{5} \in A^{*}$

- $f_{7}: w \longrightarrow w ; w \in A^{*}-\left(\operatorname{Dom}\left(\int_{1} \cup \int_{2} \cup f_{3} \cup f_{4} \cup f_{5}\right)^{4}\right.$

If we precompute the transducer representing the rational transduction $f=\left(f_{4} \circ f_{3} \circ f_{2} \circ f_{1}\right) \cup\left(f_{5} \circ\right.$ $\left.f_{6}\right) \cup f_{7}$ then the analysis of the sentence is a two-step application of $f$, namely

$f([\mathrm{P}]$ Max bouglit a little bit more than five hundred share certificates $[\mathbf{P}])=$

[N Max N] bought [N (pred a little bit more than pred) (num five hundred num) (cn share certilicates $\mathrm{cm}$ ) N]

and

$$
f^{2}([\mathbf{P}] s[\mathbf{P}])=
$$

( $\mathrm{N} \operatorname{Max} \mathrm{N}$ ) bought ( $\mathrm{N}$ a little bit more than five hundred share certificates $N$ )

which is the analysis of the sentence ${ }^{5}$.

\section{FORMAL DESCRIPTION}

\section{The algorithm}

Formally, at transducer $T$ is defined by a b-uplet $(A, Q, i, F, d, \delta)$ where $A$ is a finite alphabet, $Q$ is a finite set of states, $i \in Q$ is the initial state, $r \subset Q$ is the set of terminal states, at the transition finction maps $Q \times A$ to the set of sulsets of $Q$ and $\delta$ the emission function maps $Q \times A \times Q$ to $A$.

The core of the procedure consists in applying a transducer to a $\mathrm{PSA}$, the algorithm is well known, we give it here for the sake of readability.

is_fixed_point $=\Lambda_{\text {p p ply }}$ Transducer $\left(A_{1} T_{1}, A_{2}\right)$

$1 i=0 ; P[0]=\left(i_{1}, i_{2}\right) ; n=1 ; q=0 ; i_{-}$fixed-point $=$YES; 2 do \{

$\left.3\left(x_{1}, x_{2}\right)=P[q]\right)$

4 if $x_{1} \neq i_{1}$ then $i s_{-}$fixed-point $=N O^{\circ}$

5 if $x_{1} \in F_{1}$ and $x_{2} \in r_{2}$ then $x \in F$

\footnotetext{
${ }^{3}$ Here $f_{2}$ simmlates a context sensitive analysis becanse of $w^{\prime} \in A^{*}-\{N U M E R A L\}$

${ }^{4} \operatorname{Dom}(f)$ stands for the domain of $f$.

${ }^{5}$ Note that it is al ways possible to keep more information along the analysis and to keep track, for instance, of the position of the determiners.
} 


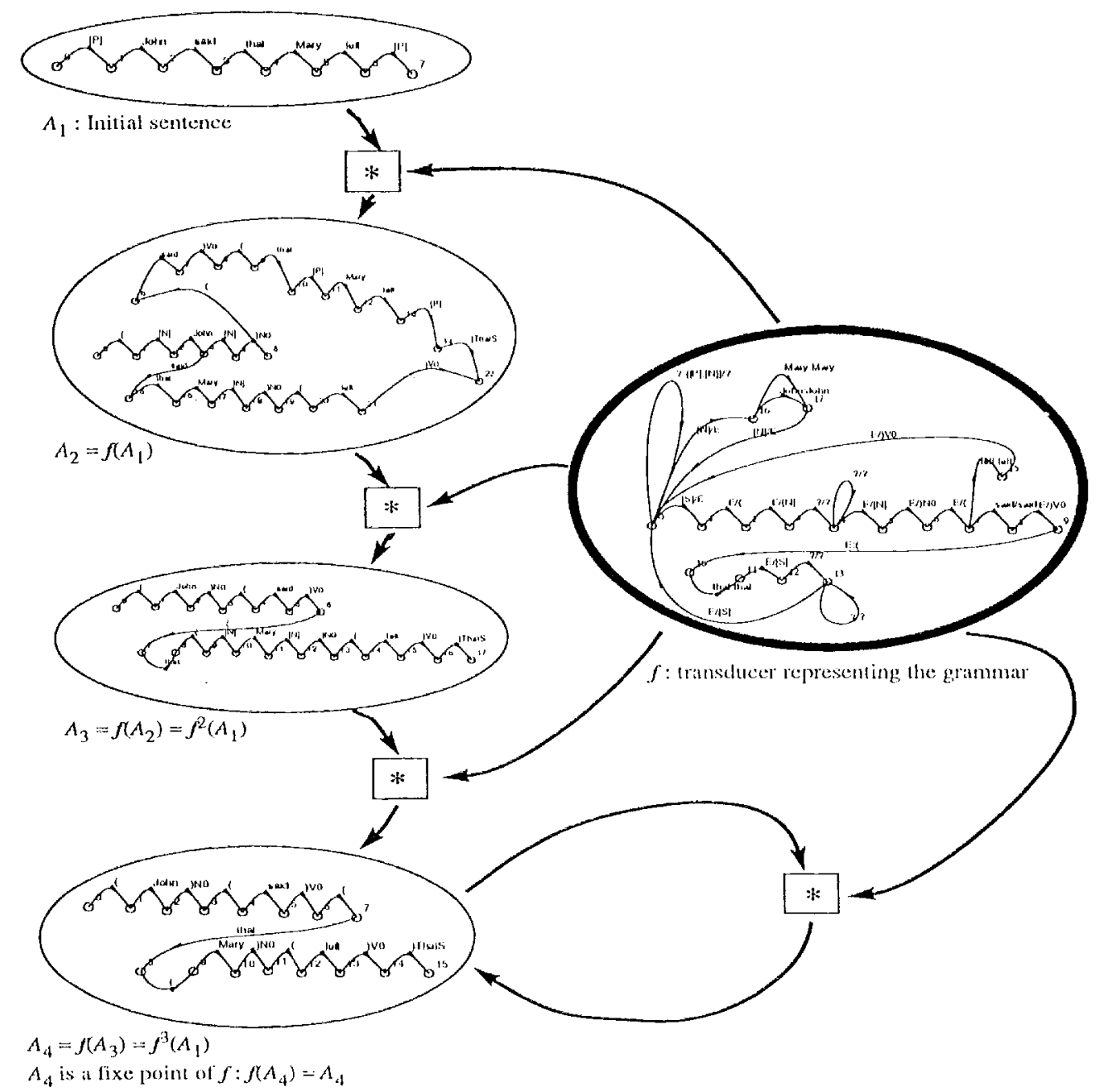

ligure 1: Overview of the analysis of the sample

; foreach $s \in A l p h \mid d_{1}\left(x_{1}, s\right) \neq \emptyset, d_{2}\left(x_{2}, s\right) \neq \emptyset$

formach $z_{1} \in d_{1}\left(x_{1}, s\right)$ and $y_{2} \in d_{2}\left(x_{2}, s\right)$

if $\exists p<n$ such that $P[p]==\left(y_{1}, y_{2}\right)$ l. hen $e=p$

clsep $P[e=n++]=\left(y_{1}, y_{2}\right)$;

add $e$ to $d\left(q, \delta_{1}\left(x_{1}, ., x_{2}\right)\right)$;

$12 \mathrm{q}^{+}+$;

133 while $(q<n)$;

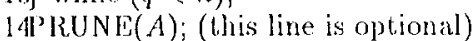

1 return is_fixed_.point;

The analysis algorithm is then the following one:

$$
\begin{array}{ll}
\text { ANALYSE_l }(A, T) & \\
1 & \text { fin }=\text { NO; } \\
2 & \text { while fin } \neq \text { Y FS do } \\
3 & \text { fin }=\text { Applyt'ransilucer }(A, T, A)
\end{array}
$$

Transducers v.s. Context Free Grammars

It should be pointed out that, given a Context-Free Crammar, it is always possible to build a transducer such that this mothod applies. In other words, any context. fres grammar can be translited into at transdueer such that the algorilhom patse the hanguage do. scribed by this grammar. Moreover, the operation that transforms a cher into its related transducer is itself a

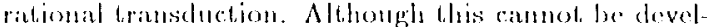
opped here due to the lack of place, this result comes uaturally when looking at the example of section 3.1 .

Moreover the method has much more expressive power than CP(i, in fact computing a fixed point of a rational transduction has the same power as applying a Thring Machine (althouglt there might not be any practical interest for that).

\section{THE SECOND ALGORTTHM : A DETFRMINISTIC DEVICE}

Given a transducer representing the grammar there al'o two diflerent ways of obtatuing new parsing programs. The lirst solution is to build a transducer ' $T$ " equivalent to ' $T$ ' from the view point of their fixed points, 
$T \sim$ fixed-point $T^{\prime}$. Namely $T \sim$ fixed-point $T^{\prime \prime}$ iff for each $x \in A^{*}, T(x)=x \Leftrightarrow T^{\prime}(x)=x$. For instance, if $T$ is such that for each $x \in A^{*}, T^{n}(x)$ converges then $T^{2} \sim$ fixed-point $T$. The second approach is to try using a different representation of $T$ or to apply it differently. In this section, we shall give an algorithm illustrating this second approach. The basic idea is to transform the finite-state transducer into a deterministic device called bimachine [1]. We will detail that latter but, basically, a bimachine stands for a left sequential function (i.e deterministic from left to right) composed to a right sequential function (i.e. deterministic from right to left). Such a decomposition always exists.

The interest of this concept appears when one looks at how the algorithm $A$ pply Transducer performs. In fact the output DAC of this algorithm has a lot of states that lead to nothing, i.e. states that are not coaccessible, thus the PRUNE function (called on line 14 of the $A$ pplyTransducer function) has to remove most of the states (around $90 \%$ in oul parser of French).

Let us for instance consider the following example: suppose the transducer $T_{a}$ is the one represented figure 2 and that we want to compute $T_{d}(A)$ where $A$ is the DAC given figure 2.

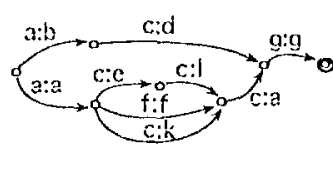

$T_{a}$

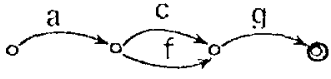

A
Pigure 2: lefl: initial transducer; right: initial DAG

Following the algorithm described in Apply'transducer up to line 14 excluded provides the DAG $A^{\prime}$ of figure 3 .

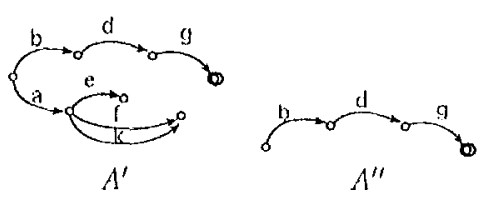

Figure 3: left: before pruning; right:after pruning

The PRUNE function has then to remove 3 of the six states to give the DAG $A^{\prime \prime}$ of figure 3

A way to avoid the overhead of computing unnecessary states is to first apply a left sequential transducer $T_{a n}$, (that is a transducer deterministic in term of its input when read from left to right) given figure 4 and then apply a right sequential transducer $l_{a b}$ (i.e. deterministic in term of its input when read from right to left) given figure 4 . We shall call the pair $B_{a}=\left(T_{a b}, T_{a b}\right)$ the bimachine functionally equivalent to $T_{a}$, i.e. $B_{a} \sim$ function $T_{a}$. With the same inpul $A$ we first obtain $A_{a}=T_{a a}(A)$ of figure 5 and then $A_{b}=$ $A^{\prime \prime}=\operatorname{reverse}\left(T_{a b}\left(\operatorname{reverse}\left(A_{a}\right)\right)\right)=T(A)=B_{a}(A)$.
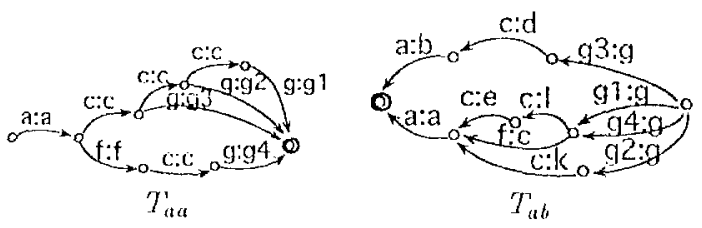

Figure 4: left:eft sequential function; right:right sequential function

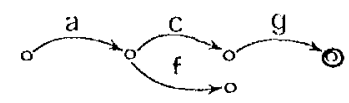

Figure 5: $A_{\text {a }}$

It should be pointed out that both $T_{a a}$ and $T_{a b}$ are deterninistic in term of their input, i.e. their left labels, which was not the case to $T_{a}$. Just like for ISA, the fact that it is deterministic implies that it can be applied faster (and sometime much faster) than nondeterministic devices, on the other hand the size of the bimachine might be, in the worst case, exponential in term of the original transducer. The following algorithm formalizes the analysis by mean of a bimachine?

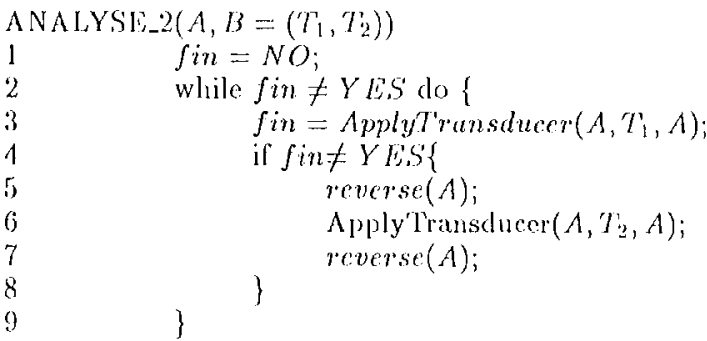

\section{IMPLEMENTATION AND RESULTS}

The main motivation for this work comes from the linguistic claim that the syntactic rules, roughly the sentence structures, are mostly lexical. 'The grammar of French we lad at our disposal wats so large that none of the available parsers could handle it.

Althongh the implemented part of the grammat is still incomplete, it almaly describes 2,878 sentential verbs (coming from [6]), that is verbs that can take a sentence as argument, leading to 201,723 lexical rules ${ }^{\text {t }}$; 1,359 intransitive verbs [2] learling to 3,153 lexical rules; 2,109 transitive verlos [3] Icading to 9,785 lexical rules; 2,920 frozen expression (coming from [7]) leading to 9,342 lexical rules and 1,213 partly frozen adverbials leading to 5,032 lexical rules. Thus, the grammar doscribes 10,479 entries and 229,035 lexical rules. This

\footnotetext{
TThe FSA reverse $(A)$ is $A$ where the transitions have been reversed and the initial and final states exchanged.

${ }^{B}$ For a verb like étonner the set of rules include Nhum élonner $N$ hum $m_{1}$ as well as $N$ hum anoir ritonné $N$ hum,

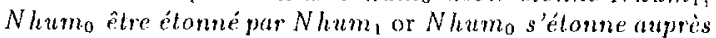
de $N h_{u m}$ de ce $Q u P_{2}$ which gives an idea of how these complexe verbs generate all average of 100 rules, or sentence structures, even if no embbeding is involved at this stige.
} 
granmar is represented by one transducer of 13,408 states and 47,119 transitions stored in 908K13.

'The following input :

Iran est agace par le fait que som ami, clans la crainte d'ôtre puni par. ses parents, ne lene ait pas avoué ses manvaises notesi.

is parsed in the following way in $0.95 \mathrm{~s}^{9}$ with a program implementing the algorithm $A N A I Y S L_{1}-1$.

(N Jean )N est \&Vppo agacé par le farit_QuP le fait: ( $Q$ ur que $(N$ son n ami ami ) N, (ADV dans la crainte de (VOW No otre deVppo puni par (N ses n parent parents ) $N$ VoW) ADV) , leur \#Nhum2 avoir ait (op \#ne-pas op) \&Vppo avoué ( $N$ ses matuvaises 11 note notes $) \mathrm{N}(\mathrm{QuP})$

'T'ypical time spending varies from 0.05 second for a ten words sentence to 5 seconds for a hundred words sentence under the current, implententation. $A$ kay point about this method is that the time spending is quite insensitive to the size of the grammat, this is crucial for scaling up the program to much larger grammars. For instance the preceding example is analyzod in $0.93 \mathrm{~s}$ (instead of $0.95 \mathrm{~s}$ ) for a grammar of half its size (around 100,000 lexical rules).

The coverage of this grammar still has lo be extended, not all data we had at our dispossal are yet encoded in the transducer (around $50 \%$ remain). 'Thus, given an arbitrary text, whereas most of the simple short sentences (five to fifteen words) are analyzed, the probability of having all lexical descriptions for longer sentences decreases rapilly. However, since all the lexical rules have been checked by hand one by one, the accuracy of the analysis is ligher than what can be expected with less lexicalized grammars. This moans two thing:s:

- whenever the analysis is found and unless the sentence is syntaclically ambiguons, the analysis is unicule,

- incorrect sentences are systematically rejected. 'lluss the set of sentence defined by the patrise: is a subset of the set of correct sentences. 'lhis property is very difficult to itcheve through nom or less lexicalized grammars.

\section{CONCLUSION}

We have introduced two diflerent parsing algorithms based on Finite-State Transducers illustrating a method capable of handling extremely large grammars very efficiently. We have shown how linite-State Transducers can handle not only finite state grammals but also hierarchical cescriptions expressed by contextfree based formalisms.

${ }^{\circ}$ On an $11 \mathrm{P} 720$, this is the nnique parsing, in other words the input is found not to be anbignous. The time spendings includes a morphological analysis by mean of at dichonary look-up. This inflected form dictionary contitins $600(1,000)$ entries [5].
'The method has been successfully implemented for a french lexicon-(irammar consisting of 200,000 lexical ruks. The use of Finite-State 'Thansducers yields a typical response time of a fractions of a second.

We have also introduced a bidirectional palsing, algorithm which further improves response lime.

'lhese investigations have, we believe, important implications for the implementalion of large grammass. Moreover, it should be possille to improve these results appreciably by exploring, different representations and diflerent decompositions of the granmar transducep with tools from the throry of Finite-State 'Transduccrs.

\section{References}

[1] Berstel, Jean, 1979. Transductions and Contextfirec Languages. Stuthgart, B.(: Teubner 277p.

[2] Boons, Jean-Panl; Alain Coullet; Ghristion Leclere 1976. La structure des phrases simples on frangats. I Constrnctions intransitines. Ciences: Dro: 377 ).

[:3] Boons, Jean. Pant; Alain Cuillev; Christian Leclere 1976. La strueture des phrases simples en

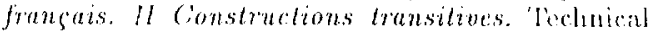
Report LADL. Universite Paris 7. Paris.

[4] Clemenceau, David; Emmantel Roche, 1993. Enhancing a morphological dictionary with two-lewel rules liACL'93, Procedings of the Conference. Utrecht.

[5] Comblois, Blandine, 1989. DHLAS : Dirlionname Silectronique du LADL pour les mols simples du frangais. Rappore technique du IAJU, Paris: Université l'aris 7

[6] Cross, Maurice, 1975. Mélhodes en syntlaze, régime des construtions complétines. Paris: Hermatu, $115 \mathrm{~s}$.

[7] Ciross, Maurice, 1986. Crammaire transformationnelle du français: 3) Syntaxe de l'udverbe. Paris: Cantiline, $669 \mathrm{p}$.

[8] Kartunen, Lauri; Ronald M. Kaplan; Annie Zachen 1992. T'mo-Lenel Morphology with Composition. Col, Ne('92, l'rocedings of the conference. Nallites.

[9] Peireira, liemando ( N. N. Rebecen N. Wright, 1991. Hinile-stale Approximation of Hortse. Structure Cirtmmars. 29th Anmual Meeting of the $\Lambda(T$, Procedings of the conference. University of California, Berkeley.

[10] Roche, Limmanuel, 1993. Analyse Syulaxique Transformationnelle du français par Thansdacteurs ot hexiqu-(mammaire. PhD dissertation, Universile Paris 7, Paris.

[11] 'lapanainen, Pasi; Atro Voutilainen, 1993. Ambiguity resolution in a reductionistic parser. Sixth Conference of the linropean (hapter of the $\mathrm{ACA}$, I'roceedings of the Conference. Utrecht, April 1993.3 